\title{
LOOKING THROUGH TRANSGENIC ZEBRAFISH TO REVEAL NOVEL MECHANISMS OF HOST-PARASITE INTERACTION: IN VIVO REAL-TIME IMAGING OF A TRYPANOSOME INFECTION
}

\author{
Maria Forlenza ${ }^{\S}$, Eva Doro, Ffion Hammond, Pei Hongyin, Geert Wiegertjes
}

Cell Biology and Immunology Group, Wageningen University, The Netherlands

Trypanosomes are unicellular flagellate protozoan parasites often with a preference for living in the blood of their hosts. Trypanosoma carassii naturally infects carp, goldfish and related species. Under normal conditions fish Trypanosomes do not cause major problems in wild stocks or aquaculture fish species, so why study them? The reason is twofold: 1) they are beautiful to look at under the microscope; 2) they can help reveal detailed immune mechanisms including, in vivo macrophage polarization, immune evasion strategies, $\mathrm{B}$ and $\mathrm{T}$ cell proliferation and memory responses. Over the past years our group has used a trypanosome infection model of carp as a tool to trigger and modulate the immune system and gain insight in fundamental protective mechanisms. To further expand our toolbox and overcome some of the limitations of the carp model, we established a trypanosome infection model of zebrafish, the little twin sister of carp. Through the use of larvae, juvenile and adult transgenic fish, we are able to: 1) visualize, in real-time, the mechanisms of parasite extravasation and leukocyte-trypanosome interaction; 2) visualize the kinetics of innate immune cell activation by using Tnfa:Gfp and Illb:Gfp expressing transgenic zebrafish lines; and 3) investigate the role of macrophages in the resolution or exacerbation of infection through ablation experiments. Where the large carp is perfect for cellular studies, the small zebrafish is an excellent tool for live imaging and genetic manipulation. Together, they form a perfect 'twinning team' to address fundamental questions on the evolution of the immune system and to unravel fundamental aspects of host-pathogen interaction.

\section{Keywords:}

host-pathogen interaction, zebrafish, trypanosomes, immune response

\footnotetext{
${ }^{\S}$ Corresponding author. Tel.: 0031317483336

E-mail address: maria.forlenza@wur.nl
} 